\title{
Computable Finite Element Error Bounds for Poisson's Equation
}

\author{
P. ARBenz \\ Institut fur Angewandte Mathematik, \\ Universität Zürich, \\ Rämistrasse 74, 8001 Zürich, Switzerland
}

[Received 26 May 1982]

New explicit finite element error bounds are presented for approximation by

(1) piecewise linear elements over triangles and

(2) piecewise bilinear elements over squares and rectangles.

By this the error bounds given in Barnhill, Brown \& Mitchell (1981) are improved.

\section{Introduction}

LET $G \subset \mathbb{R}^{2}$ be an open and bounded region with polygonal boundary $\partial G$ and let $f \in L_{2}(G)$. We consider the elliptic boundary value problem

$$
\begin{gathered}
\int_{G}[\nabla u(x, y) \cdot \nabla v(x, y)-f(x, y) v(x, y)] d x d y=0, \\
\forall v \in H_{0}^{1}(G), \quad u \in H_{0}^{1}(G),
\end{gathered}
$$

which is the weak form of the classical boundary value problem

$$
\begin{array}{rlrl}
\Delta u(x, y)+f(x, y) & =0 & & (x, y) \in G \\
u(x, y)=0 & & (x, y) \in \partial G .
\end{array}
$$

The solution $u$ of (1.1) is known to be in $H_{0}^{1}(G)$ (Ciarlet, 1978). If $G$ is convex, Barnhill \& Wilcox (1977) have shown that $u \in H^{2}(G)$ and that the inequality

holds, where

$$
|u|_{2 G} \leqslant|f|_{0, G}:=\|f\|_{L_{1}(G)}
$$

$$
|u|_{2, G}^{2}:=\int_{G}\left[\partial_{x x} u(x, y)^{2}+2 \partial_{x} u(x, y)^{2}+\partial_{y} u(x, y)^{2}\right] d x d y .
$$

We will solve the problem (1.1) by the finite element method (Schwarz, 1980): the region $G$ is subdivided in triangles or in squares (if possible). The finite element space $V_{h} \subset H_{0}^{1}(G)$ consists of continuous functions which are piecewise linear or bilinear polynomials in the triangles or squares, respectively. (The index $h$ in $V_{h}$

$$
\text { 4. } 1982 \text { Academic Press Inc. (London) Limited }
$$


indicates a measure of the fineness of the subdivisions of $G$.) The problem (1.1) is solved in $V_{k}$ giving a finite element solution $U$ which satisfies (Strang \& Fix, 1973)

$$
|u-U|_{1, G}^{2}:=\int_{G}|\nabla(u-U)(x, y)|^{2} d x d y \leqslant|u-\tilde{U}|_{1, G}^{2}, \quad \forall \tilde{U} \in V_{k} .
$$

In this paper new error bounds are derived for $|u-U|_{1, G}$ and for $|u-U|_{0, G}$ improving the bounds given in Barnhill et al. (1981).

\section{Error Bound for Triangular Elements}

The linear interpolant on the triangle $T$ with vertices $(0,0),(1,0)$ and $(0,1)$ is

$$
Q_{T} u(x, y):=(1-x-y) u(0,0)+x u(1,0)+y u(0,1), \quad u \in C(\bar{X}) .
$$

We define the interpolation error function by

$$
e_{u}(x, y):=u(x, y)-Q_{T} u(x, y) .
$$

Barnhill et al. (1981) gave the $H^{1}$-bound

$$
\left|e_{u}\right|_{1, T} \leqslant 1 \cdot 207|u|_{2 . T}, \quad u \in H^{2}(T) .
$$

This bound can be improved. Consider an arbitrary $u \in H^{2}(T)$. By Sobolev's imbedding theorem (Ciarlet, 1978) $u \in C(\bar{T})$. so that $Q_{T} u$ is defined. As all second derivatives of $Q_{T} u$ vanish, we have

$$
\left|e_{k}\right|_{2, T}=\left|u-Q_{T} u\right|_{2, T}=|u|_{2, T} .
$$

$|e|_{1}$ and $|e|_{2}$ are norms on the error functions space

$$
E=\left\{e \in H^{2}(T) \mid e(0,0)=e(1,0)=e(0,1)=0\right\} .
$$

With (2.3) we find that

$$
R[e]=\frac{|e|_{2, T}^{2}}{|e|_{1, T}^{2}}, \quad e \in E \backslash\{0\}
$$

is bounded below by a positive constant (i.e. $1 \cdot 207^{-2}$ ). We consider (2.5) as the Rayleigh quotient of the eigenvalue problem whose variational form is:

find $(i, v) \in \mathbb{R} \times(E \backslash\{0\})$ such that

$$
\begin{aligned}
& \int_{T}\left[\partial_{x x} v \partial_{x x} w+2 \partial_{x y} v \partial_{x y} w+\partial_{y} v \partial_{y} w\right] d x d y- \\
& \lambda \int_{T}\left[\partial_{x} v \partial_{x} w+\partial_{y} v \partial, w\right] d x d y=0, \quad \forall w \in E .
\end{aligned}
$$

Applying Green's formula, we obtain from (2.6) for sufficiently smooth $v$ and $w$

$$
\int_{T}\left(\Delta^{2} v+\lambda \Delta v\right) w d x d y-\int_{\Gamma T}\left(\partial_{n} \Delta v+\lambda \partial_{n} v\right) w d s+\int_{\lambda T} \partial_{n \pi} v \partial_{n} w d s+\int_{\lambda T} \partial_{n t} v \partial_{T} w d s=0 .
$$


On the edges of the triangle $T d t=d s$, thus

$$
\int_{\partial T} \partial_{m} v \partial_{1} w d s=-\int_{\partial T} \partial_{t m t} v w d s,
$$

because $w$ vanishes in the comers of $T$.

Putting (2.8) into (2.7) we obtain the classical form of (2.6)

$$
\begin{aligned}
\Delta^{2} v(x, y) & =-\lambda \Delta v(x, y) & & (x, y) \in T \\
v(0,0)=v(1,0)=v(0,1) & =0 & & \\
\partial_{n z} v(x, y) & =0 & & (x, y) \in \partial T \\
\partial_{n} \Delta v(x, y)+\partial_{\operatorname{ma}} v(x, y)+\lambda \partial_{n} v(x, y) & =0 & & (x, y) \in \partial T .
\end{aligned}
$$

With the first eigenvalue $\mu_{1}$ of (2.9) we have

$$
|e|_{1, T} \leqslant \mu_{1}^{-1}|e|_{2, T}, \quad \forall e \in E .
$$

Equality holds if $e=v_{1}, v_{1}$ being the first eigenfunction of (2.9). In this sense (2.7) is optimal. $\mu_{1}$ was computed to a high precision using $C^{1}$-finite elements. We could achieve the inclusion

$$
4 \cdot 18673 \leqslant \mu_{1} \leqslant 4 \cdot 18674 \text {. }
$$

With this $(2.10)$ becomes

$$
\left|u-Q_{T} u\right|_{1, T} \leqslant 0-4888|u|_{2 . T} .
$$

Transformation on the triangle $T_{h}$ with vertices $(0,0),(h, 0),(0, h)$ gives

$$
\left|u-Q_{T_{u}} u\right|_{1, T_{u}} \leqslant 0-4888 h|u|_{2 . T_{u}} \text {. }
$$

\section{Error Bound for Square Elements}

The bilinear interpolant on the square $S$ with vertices $(0,0),(1,0),(1,1)$ and $(0,1)$ is defined by

$$
\begin{aligned}
& Q_{s} u(x, y)=(1-x)(1-y) u(0,0)+(1-x) y u(1,0)+ \\
& x(1-y) u(0,1)+x y u(1,1), \quad u \in C(\bar{S}) .
\end{aligned}
$$

We define the interpolation error function by

$$
e_{u}(x, y)=u(x, y)-Q_{s} u(x, y)
$$

and the space of all possible error functions obtained by interpolating functions in $H^{2}(S)$ by

$$
E=\left\{v \in H^{2}(S) \mid v(0,0)=v(1,0)=v(0,1)=v(1,1)=0\right\} .
$$

Barnhill et al. (1981) gave the error bound

$$
\left|e_{\mathrm{u}}\right|_{1 . s} \leqslant 0-625|u|_{2,5}, \quad u \in H^{2}(S) .
$$

As in the former section this bound can be improved. Consider an arbitrary 
$u \in H^{2}(S) . Q_{s} u$ is again defined. Here $\left|Q_{s} u\right|_{2.5} \neq 0$ due to the $x y$-terms in (3.1)! But $\partial_{x,} Q_{s} u(x, y)$ is a constant and because

$$
\begin{aligned}
\int_{0}^{1} \int_{0}^{1} \partial_{x y} e(x, y) d y d x & =\int_{0}^{1}\left[\partial_{x} e(x, 1)-\partial_{x} e(x, 0)\right] d x \\
& =e(1,1)-e(0,1)-e(1,0)+e(0,0)=0, \quad \forall e \in E,
\end{aligned}
$$

it follows that

$$
\begin{aligned}
|u|_{2, s}^{2} & =\left|e_{x}+Q_{s} u\right|_{2, s}^{2} \\
& =\int_{0}^{1} \int_{0}^{1}\left[\partial_{x x} e_{u}^{2}+2\left(\partial_{x y} e_{x}+\partial_{x y}, Q_{s} u\right)^{2}+\partial_{y} e_{u}^{2}\right] d x d y \\
& =\left|e_{u}\right|_{2, s}^{2}+4 \int_{0}^{1} \int_{0}^{1} \partial_{x y} e_{u} \partial_{x y} Q_{s} u d x d y+2 \int_{0}^{1} \int_{0}^{1} \partial_{x y} Q_{s} u^{2} d x d y \\
& =\left|e_{w}\right|_{2, s}^{2}+\left|Q_{s} u\right|_{2, s}^{2} \geqslant\left|e_{u}\right|_{2, s}^{2}, \quad \forall u \in H^{2}(S) .
\end{aligned}
$$

Therefore we can argue as in Section 2 and compute a new bound by minimizing the Rayleigh quotient

$$
R[e]:=\frac{|e|_{2, s}^{2}}{|e|_{1, s}^{2}}
$$

in $E\{0\}$. The minimum $\mu_{1}=\pi^{2}$ is attained for functions in the two-dimensional subspace of $E$ spanned by $\sin \pi x$ and $\sin \pi y$. With this and the use of (3.5) we obtain the inequality

$$
\begin{aligned}
\left|u-Q_{s} u\right|_{1, s} & \leqslant 0-3184\left|u-Q_{s} u\right|_{2, s} . \\
& \leqslant 0-3184|u|_{2, s}, \quad \forall u \in H^{2}(S) .
\end{aligned}
$$

Transformation on the square $S_{h}=(0, h) \times(0, h)$ gives

$$
\left|u-Q_{s_{1}} u\right|_{t . s_{\mathbf{t}}} \leqslant 0-3184 h|u|_{2 . s_{i}} .
$$

Remark: For a rectangle with sidelengths $h_{1}$ and $h_{2}>h_{1}$ (3.7) holds with $h=h_{2}$.

\section{Numerical Results}

The new bounds (2.12) and (3.7) are compared with the bounds in Barnhill et al. (1981) and with the numerical results given therein. The three test problems considered there have known solutions. Therefore the theoretical (upper) bounds can be compared with corresponding computable values (Barnhill et al., 1981)

$$
K_{M}^{p}=\frac{\left|u-U_{M}\right|_{2-p}}{h^{\top}|f|_{0}}, \quad p=1,2 ; \quad M=S, T,
$$

where $u$ is the actual solution and $U_{M}$ its approximant in a finite element space of mesh width $h$ consisting of triangular $(M=T)$ or square $(M=S)$ elements. Each test problem was solved with three different mesh sizes $h$ giving nine numbers $K_{M}^{p}$ for each pair $(p, M)$. The results are summarized in Table 1 .

The bounds

$$
\left|u-U_{T}\right|_{0 . \tau} \leqslant 0.2389 h^{2}|f|_{0}
$$


TABLE 1

\begin{tabular}{ccc}
\hline Range of computable values $K_{\text {p }}^{p}$ & Bounds in Barnhill et al. (1981) & Our bounds \\
\hline $0.1489 \leqslant K_{\$}^{1} \leqslant 0.2519$ & 0.7906 & 0.3184 \\
$0-0405 \leqslant K_{S}^{2} \leqslant 0.0761$ & $0-6250$ & 0.1014 \\
$0-1517 \leqslant K_{T}^{1} \leqslant 0.3466$ & 1.207 & 0.4888 \\
$0-0417 \leqslant K_{T}^{2} \leqslant 0-1333$ & 1.457 & $0-2389$ \\
\hline
\end{tabular}

and

$$
\left|u-U_{s}\right|_{0, s} \leqslant 0-1014 h^{2}|f|_{0}
$$

are obtained from (2.12) and (3.7) using the so-called Nitsche trick (Ciarlet, 1978).

\section{Concluding Remarks}

The error bounds seem to be useful for problems of the form (1.1) on convex domains $G$.

For the three test problems the theoretical bounds are near to the greatest values of $K_{M}, p=1,2, M=S, T . K_{T}^{1}$ and $K_{S}^{1}$ are overestimated by factors from $1 \cdot 2$ to $3 \cdot 2, K_{T}$ and $K_{s}$ by factors from $1 \cdot 3$ to $5 \cdot 7$. So the upper bounds are of the correct order. The bounds are better for the energy norm than for the $L_{2}$-norm which is clear because the $L_{2}$-norm bounds have been obtained only indirectly.

In another approach to Poisson's equation Kuttler (1979) gives the inequality

$$
|u-\psi|_{0, G} \leqslant c_{1}|f-\Delta \psi|_{0, G}+c_{2}|\psi|_{0, i G}
$$

and determines the constants $c_{1}$ and $c_{2}$ explicitly. Here $u$ is the solution of (1.2), $\psi$ an arbitrary approximation in the domain of $\Delta$. This inequality is not applicable on finite element approximations because piecewise linear or bilinear functions are not elements of the domain of the Laplace operator.

\section{REFERENCES}

BarnhilL, R. E. \& WILCOX, C. H. 1977 Computable error bounds for finite element approximations to the Dirichlet problem. University of Utah report.

BARNHILL, R. E., Brown, J. H. \& MITCHELI, A. R. 1981 A comparison of finite element ertor bounds for Poisson's equation. IMA J. Num. Analysis 1, 95-103.

Ciarlet, P. G. 1978 The Finite Element Method for Elliptic Problems. Amsterdam, New York, Oxford: North-Holland.

KUTTLER, J. R. 1979 Dirichlet eigenvalues. SIAM J. Num. Analysis 16, 332-338.

SCHWARZ, H. R. 1980 Methode der finiten Elemente. Stuttgart: Teubner.

Strang, G. \& FDx, G. 1973 An Analysis of the Finite Element Method. Englewood Cliffs, N.J.: Prentice-Hall. 